PALABRAS CLAVE

Desarrollo rural

Mitigación de la pobreza

Zonas rurales

Política de desarrollo

Gastos públicos

Descentralización gubernamental

Gobernabilidad

Indicadores económicos

Guatemala
Ottoniel Monterroso-Rivas

Investigador Dedicación Completa,

Instituto de Agricultura, Recursos

Naturales y Ambiente (IARNA),

Universidad Rafael Landívar,

Guatemala

- aomonterroso@url.edu.gt
REVISTA CEPAL 97 - ABRIL 2009

\section{Institucionalidad y políticas públicas para el desarrollo rural en Guatemala}

\author{
Ottoniel Monterroso-Rivas
}

$\mathrm{E}$

n este artículo se revisa la institucionalidad formal para la aplicación de las políticas de desarrollo rural en Guatemala, que derivó de la modernización del Estado promovida mediante los Acuerdos de Paz. La tesis principal es que las políticas de desarrollo rural serán más eficientes si se fundamentan en la institucionalidad a que dichos acuerdos dieron lugar, en la que se diferencian tres niveles de gobierno: central, desconcentrado y descentralizado. En los dos últimos se ejecuta el 43\% de la inversión pública total. El gobierno central debería focalizar el presupuesto en los territorios pobres, disminuir el gasto en subsidios privados y aumentar la provisión de bienes públicos. En cuanto a los niveles desconcentrado y descentralizado, se ha demostrado la eficacia del enfoque territorial como medio para la generación de políticas, pero es preciso que la planificación territorial se realice a partir de pactos político-sociales entre los actores locales. 


\section{I}

\section{Introducción}

El debate reciente sobre las políticas de reducción de la pobreza en Guatemala se ha enfocado en el desarrollo rural y en la necesidad de implementar medidas específicas para ello. Esta atención obedece a que las áreas rurales concentran la mayor proporción de población que vive en la pobreza y la indigencia (INE, 2007), pero también a los efectos poco significativos de las políticas sociales y de apertura comercial en materia de disminución de los índices de pobreza en el país (Banco Mundial, 2003 y 2004).

Las políticas de desarrollo rural en Guatemala se han originado principalmente a partir de fuentes internas y externas. Entre las primeras se cuentan los Acuerdos de Paz firmados en 1996, de los cuales derivaron acciones específicas orientadas a las zonas rurales. Los convenios relacionados con el desarrollo rural fueron el Acuerdo sobre Aspectos Socioeconómicos y Situación Agraria, el Acuerdo para el Reasentamiento de las Poblaciones Desarraigadas por el Conflicto Armado y el Acuerdo sobre Identidad y Derechos de los Pueblos Indígenas, que ordenaron la creación de nuevas instituciones públicas para impulsar la formulación de políticas relacionadas con temas agrarios, agrícolas y rurales.

En cuanto a las fuentes externas, al comienzo predominaron las políticas de ajuste estructural y de apertura comercial cuyo objetivo era fomentar el crecimiento en las áreas rurales. Sin embargo, recientemente se han incorporado a la discusión el sistema de fincas en su versión actualizada de medios de vida sostenibles (Ellis, 2000) y el enfoque territorial, derivado este último de

\footnotetext{
$\square$ El autor agradece el apoyo financiero de la Organización de las Naciones Unidas para la Agricultura y la Alimentación (FAO), representación en Guatemala, para la elaboración del presente artículo. Este se basa en los resultados del Proyecto de apoyo a la institucionalización de las políticas de desarrollo rural en Guatemala, Convenio de Cooperación USAID/IICA No. 520-A-00-05-00096-00 (IICA/USAID, 2008). También agradece los comentarios de Magali Quintana, Juan Carlos Méndez, Juventino Gálvez, Jaime Carrera, Magaly Arrecis y un examinador anónimo a las versiones preliminares. Las observaciones e ideas expresadas en el documento son de su exclusiva responsabilidad y no representan el punto de vista de la FAO, el Instituto Interamericano de Cooperación para la Agricultura (IICA) o la Agencia de los Estados Unidos para el Desarrollo Internacional (USAID).
}

la experiencia europea en materia de desarrollo rural (Sepúlveda y otros, 2003).

Si bien el debate interno y externo sobre el desarrollo rural ha contribuido a definir "qué" políticas deben adoptarse en este campo, aún existe un vacío conceptual sobre la institucionalidad necesaria para llevar a cabo las acciones identificadas. La pregunta ahora es "cómo" ordenar a los actores públicos, sociales y privados para promover el desarrollo de las áreas rurales, de manera que en la discusión actual ha adquirido relevancia el tema de los arreglos institucionales. Las políticas públicas de desarrollo rural requieren de una institucionalidad dinámica, capaz de implementar las medidas pertinentes y que aborde el problema desde una perspectiva multisectorial, con un enfoque territorial, descentralizado y de participación ciudadana. En otras palabras, se necesita un Estado moderno que pueda llevar a la práctica las orientaciones de política.

El objetivo de este artículo es revisar la institucionalidad formal derivada de los Acuerdos de Paz para la modernización del Estado guatemalteco, discutiendo los aspectos relevantes de las políticas de desarrollo rural. La tesis principal es que estas pueden impulsarse eficazmente sobre la base de esa institucionalidad, puesto que permitirá inyectarle mayor sostenibilidad a las acciones y contribuirá al fortalecimiento de las entidades del Estado. De esta forma, las políticas de desarrollo rural pueden convertirse en un instrumento de consolidación de la institucionalidad estatal del país.

Para ello, en la primera sección se analizan las orientaciones de política que se han impulsado en Guatemala desde la firma de los Acuerdos de Paz. En este apartado se revisa el gasto público rural de los gobiernos central, desconcentrado y descentralizado, así como los logros y repercusiones de las políticas pertinentes en el período 1996-2007. Se resumen luego las discusiones locales sobre el desarrollo rural, seguido de una descripción de la estructura institucional formal existente - es decir, la respaldada por un marco legal específico- - para fomentar el desarrollo de las zonas rurales. En la quinta sección se señalan los principales aspectos de esa institucionalidad que requieren de fortalecimiento $\mathrm{y}$, finalmente, se exponen las conclusiones más importantes. 


\section{II}

\section{Políticas de desarrollo rural en Guatemala en el período 1996-2007}

\section{Las orientaciones de política}

El Acuerdo de Paz Firme y Duradera suscrito en 1996 marcó un cambio de dirección en materia de políticas de desarrollo rural en Guatemala. Por una parte, se reconoció que era preciso superar las condiciones de pobreza, desigualdad y marginación social y política que habían sido los principales obstáculos para el desarrollo integral del país y fuente de la conflictividad político-militar. Por otra, se planteó que para lograr este objetivo era necesario fortalecer la democracia real, funcional y participativa y que el proceso de desarrollo económico y social implicaba la concertación de las políticas públicas entre los actores sociales y el Gobierno (Gobierno de Guatemala/Unidad Revolucionaria Nacional Guatemalteca, 1996).

La agenda rural enmarcada en los Acuerdos de Paz comprende elementos de carácter social, económico y agrario. En el programa social se incluyeron medidas relacionadas con la provisión de salud, educación y otros servicios básicos a la población rural. La agenda económica se centró en el fomento de la competitividad rural, incluidos los aspectos de comercio exterior, acceso a los activos de producción y aumento de la inversión pública en las áreas rurales. En cuanto al programa agrario, se planteó la necesidad de abordarlo a partir de mecanismos de mercado, generando instrumentos de política tales como i) proporcionar certeza jurídica sobre los derechos de propiedad de la tierra, ii) evitar la concentración de la tenencia de la tierra mediante un impuesto territorial a los latifundios y tierras ociosas, iii) establecer un marco legal para la discusión y solución de conflictos agrarios y iv) crear condiciones favorables para el acceso a la propiedad de la tierra de los grupos campesinos.

Al mismo tiempo que se promovía la agenda de la Paz, a partir de 1996 se impulsaron activamente políticas de desregulación del Estado, ajuste estructural y apertura comercial. Entre otras medidas, se privatizaron activos y se rebajó la carga fiscal del Ministerio de Agricultura, Ganadería y Alimentación, lo que implicó una reducción de los instrumentos intervencionistas y del número de funcionarios. También se fomentó el comercio internacional mediante tratados comerciales, lo que se tradujo en nuevos mercados pero, a su vez, en una mayor competencia interna.

\section{El gasto público rural}

El Estado guatemalteco ha implementado dichas políticas a lo largo de tres períodos de administración pública, con diferentes grados de avance. ${ }^{1}$ Por ejemplo, a partir de 1996 se amplió el gasto público social en las áreas rurales y se incrementó considerablemente la inversión pública en infraestructura. En el sector agrario, se modernizó el Registro General de la Propiedad y se creó el Registro de Información Catastral, centrados en la certeza jurídica sobre la propiedad de la tierra. También se formaron la Secretaría de Asuntos Agrarios, orientada a la resolución de conflictos, y el Fondo de Tierras como mecanismo transitorio para facilitar el acceso a la tierra. ${ }^{2}$ En el ámbito productivo, se ampliaron las inversiones gubernamentales en las zonas rurales, se transformó al Banco de Desarrollo Rural en una sociedad anónima de capital mixto y se potenciaron los acuerdos de libre comercio.

Un análisis del gasto público en las zonas rurales permite un acercamiento a lo que han sido las políticas de desarrollo pertinentes. El cuadro 1 muestra que tras la firma de la paz, en 1996, dicho gasto se incrementó a una tasa media anual del $11 \%$ en términos reales. A partir de esa fecha se le dio mayor importancia a la infraestructura rural, que en el período presidencial 1996-1999 llegó a representar hasta el 60\% del gasto público total del gobierno central. En el último período analizado (2004-2006) la inversión en infraestructura representó un promedio del $40 \%$ y el gasto en política social ascendió al 50\% del total. Por su parte, la participación del fomento productivo se ha mantenido cercana al $11 \%$ del gasto público total en el sector rural. Las metas de

\footnotetext{
${ }^{1}$ Para una revisión del cumplimiento de los Acuerdos de Paz véanse, por ejemplo, MINUGUA (2004) y Gobierno de Guatemala/Secretaría de la Paz (2007).

${ }^{2}$ Aún están pendientes el impuesto territorial y la creación de los tribunales agrarios, instrumentos clave para dinamizar los mercados de la tierra.
} 
Composición del gasto público rural del gobierno central, 1985-2006

(En millones de quetzales de 2000)

\begin{tabular}{|c|c|c|c|c|}
\hline Año & Gasto social & Infraestructura & Fomento productivo & Total \\
\hline 1985 & 604 & 827 & 256 & 1687 \\
\hline 1986 & 545 & 509 & 477 & 1531 \\
\hline 1987 & 620 & 1174 & 441 & 2235 \\
\hline 1988 & 738 & 718 & 627 & 2083 \\
\hline 1989 & 761 & 626 & 569 & 1957 \\
\hline 1990 & 573 & 565 & 333 & 1472 \\
\hline 1991 & 633 & 727 & 319 & 1679 \\
\hline 1992 & 268 & 535 & 348 & 1151 \\
\hline 1993 & 736 & 1040 & 301 & 2078 \\
\hline 1994 & 736 & 707 & 296 & 1739 \\
\hline 1995 & 723 & 1375 & 259 & 2356 \\
\hline 1996 & 1262 & 1717 & 188 & 3167 \\
\hline 1997 & 999 & 2439 & 294 & 3731 \\
\hline 1998 & 1616 & 2446 & 439 & 4500 \\
\hline 1999 & 1040 & 3854 & 683 & 5577 \\
\hline 2000 & 1496 & 1803 & 432 & 3731 \\
\hline 2001 & 1455 & 1677 & 837 & 3969 \\
\hline 2002 & 1466 & 2946 & 691 & 5103 \\
\hline 2003 & 1479 & 2094 & 555 & 4127 \\
\hline 2004 & 2590 & 2210 & 517 & 5317 \\
\hline 2005 & 3095 & 2130 & 794 & 6019 \\
\hline 2006 & 3532 & 2832 & 790 & 7155 \\
\hline
\end{tabular}

Fuente: para datos del período 1985-2001: Kerrigan, G., Gasto público hacia el sector agrícola y desarrollo de las áreas rurales: ALC, tendencias y desafíos, Santiago de Chile, Organización de las Naciones Unidas para la Agricultura y la Alimentación (FAO), 2001; para 2002-2004: Instituto Interamericano de Cooperación para la Agricultura/Agencia de los Estados Unidos para el Desarrollo Internacional (IICA/USAID), "Actualización de la base de datos del gasto público rural del período 2002-2006", Informe de consultoría, IICA-Guatemala, 2007; para 2004-2006: Secretaría de Planificación y Programación (SEGEPLAN), “Actualización de la base de datos del gasto público rural del período 2006-2007”, Informe de consultoría, Ciudad de Guatemala, 2008.

los Acuerdos de Paz en materia de gasto público social e inversión en infraestructura se han sobrepasado al menos un $50 \%$ en los diversos presupuestos del período 1996-2007 (SEGEPLAN, 2008a).

La serie histórica de la composición del gasto público rural se estimó siguiendo la metodología de la Organización de las Naciones Unidas para la Agricultura y la Alimentación (FAO) (Kerrigan, 2001), en que el gasto se divide en más de 25 partidas. Sin embargo, en los últimos diez años (1996-2006) el 91\% del gasto rural de Guatemala se ha dedicado a seis rubros específicos: obras viales $(33 \%)$, educación rural $(28 \%)$, fomento productivo agrícola (10\%), infraestructura social (10\%), salud y nutrición rural $(6 \%)^{3}$ y emisión de títulos de compra de tierras $(4 \%)$.

La estructura del gasto rural de Guatemala a partir de 1996 siguió el mismo patrón de América Latina (Fan y Rao, 2003), en que el gasto social ha sido el principal

\footnotetext{
${ }^{3}$ La participación del gasto en salud y nutrición aumentó un $11 \%$ en los últimos cuatro años y explica el alza del gasto social que se muestra en el cuadro 1.
}

instrumento de mitigación de la pobreza, mientras que las inversiones en infraestructura y fomento productivo se orientaron a la promoción de las exportaciones, principalmente en los sectores que tienen ventajas competitivas.

El desarrollo rural también se ha visto favorecido por las políticas de descentralización del Estado, ya que los recursos presupuestarios de los gobiernos departamentales y municipales son una fuente importante de financiamiento. En 2007 la inversión pública impulsada a nivel departamental y municipal representó el $43 \%$ del total nacional (véase el cuadro 2). Ese año las inversiones ejecutadas por las tres instancias de gobierno ascendieron a 1.428 millones de dólares.

En el cuadro 3 se muestran las inversiones realizadas por los gobiernos departamentales en el sector rural durante el período 2004-2007, a partir de las transferencias del gobierno central a los consejos departamentales de desarrollo (CODEDE). En esos cuatro años la inversión rural a nivel de departamentos fue de 2.714 millones de quetzales (aproximadamente 362 millones de dólares) y representó el $74 \%$ del monto total de recursos recibidos por los gobiernos pertinentes. 
CUADRO 2

Inversión pública ejecutada en los tres niveles de gobierno, año 2007

(En millones de quetzales y dólares ${ }^{\mathrm{a}}$ )

\begin{tabular}{lccr}
\hline Tipo de gobierno & Quetzales & Dólares & Porcentajes \\
\hline Gobierno central & 5838 & 761 & 53 \\
Instancias autónomas & 461 & 60 & 4 \\
Gobierno desconcentrado (departamental) & 1067 & 139 & 10 \\
Gobierno descentralizado (municipal) & 3587 & 468 & 33 \\
Total & 10953 & 1428 & 100
\end{tabular}

Fuente: Secretaría de Planificación y Programación (segePlan), Avances y desafíos de las políticas públicas en la administración Berger: ejercicio de transición, Ciudad de Guatemala, 2008.

a Al tipo de cambio medio anual de 7,67 quetzales por dólar (véase Banco de Guatemala, "Indicadores económicos de Guatemala” [en línea] http://www.banguat.gob.gt/).

b Se refiere a los aportes del gobierno central a los consejos departamentales de desarrollo; no incluye las inversiones realizadas por los ministerios en forma desconcentrada ni los fondos de inversión social.

CUADRO 3

Composición de las inversiones realizadas en áreas rurales mediante el aporte a los consejos departamentales de desarrollo, 2004-2007 (En miles de quetzales corrientes)

\begin{tabular}{|c|c|c|c|c|}
\hline \multirow{2}{*}{ Tipo de inversión } & \multicolumn{4}{|c|}{ Proyectos aprobados } \\
\hline & Quetzales & Porcentaje & Número & Porcentaje \\
\hline \multicolumn{5}{|l|}{ Infraestructura } \\
\hline Caminos y carreteras & 920365226 & 43 & 2500 & 47 \\
\hline Agua y saneamiento & 741770584 & 35 & 1702 & 32 \\
\hline Desarrollo urbano & 401777511 & 19 & 950 & 18 \\
\hline Energía & 27443119 & 1 & 67 & 1 \\
\hline Vivienda & 15437009 & 1 & 44 & 1 \\
\hline Otros infraestructura & 24915924 & 1 & 84 & 2 \\
\hline Total infraestructura & 2131709373 & $79^{\mathrm{a}}$ & 5347 & $72,5^{\mathrm{a}}$ \\
\hline \multicolumn{5}{|l|}{ Inversión social } \\
\hline Educación & 470073731 & 89 & 1702 & 90 \\
\hline Salud y asistencia social & 41083590 & 8 & 136 & 7 \\
\hline Cultura y deportes & 8549874 & 2 & 27 & 1 \\
\hline Otros gasto social & 5739234 & 1 & 16 & 1 \\
\hline Total inversión social & 525446428 & $19^{\mathrm{a}}$ & 1881 & $25,5^{\mathrm{a}}$ \\
\hline \multicolumn{5}{|l|}{ Fomento productivo } \\
\hline Agropecuario & 47203009 & 83 & 128 & 86 \\
\hline Industria y comercio & 5427217 & 10 & 8 & 5 \\
\hline Organización productiva & 2333541 & 4 & 4 & 3 \\
\hline Ciencia y tecnología & 1486154 & 3 & 4 & 3 \\
\hline Otros fomento productivo & 506224 & 1 & 4 & 3 \\
\hline Total fomento productivo & 56956145 & $2^{\mathrm{a}}$ & 149 & $2^{\mathrm{a}}$ \\
\hline Gran total & 1164805147 & 323 & 4059 & 226 \\
\hline
\end{tabular}

Fuente: elaboración propia sobre la base de Secretaría de Planificación y Programación (SEGEPLAN), "Análisis del comportamiento del aporte a los consejos departamentales de desarrollo en el marco de las orientaciones de política período 2004-2007”, Dirección de Inversión Pública, Ciudad de Guatemala, 2007, inédito.

a Cifras expresadas como porcentajes del gran total. 
La estructura de la inversión departamental continuó orientándose significativamente a la infraestructura (79\% del total), en que los caminos - construcción, mantenimiento y ampliación- representaron el 43\% del total. En el rubro de inversión social de este nivel de gobierno se incluyen los proyectos cuya finalidad es de índole social, pero en que predominan las obras de infraestructura, tales como construcción y remodelación de escuelas (89\%), construcción y remodelación de centros de salud (8\%) y construcción de canchas deportivas (2\%).

Algunos ejemplos específicos de proyectos emprendidos por los consejos departamentales son el adoquinamiento de calles, la remodelación de parques, mercados o edificios públicos y la construcción de salones comunales y de canchas deportivas. Esto no solo demuestra la falta de coordinación con las políticas del gobierno central, sino que plantea serias dudas sobre la eficiencia y eficacia de la inversión pública realizada por este nivel de gobierno en materia de disminución de la pobreza.

Los gobiernos departamentales destinaron únicamente el $2 \%$ del total de recursos al fomento productivo (56 millones de quetzales durante el período 2004-2007), siendo el sector agropecuario el que absorbió más del $83 \%$ de ellos. Entre los proyectos del caso destacan los forestales, de riego y diferentes iniciativas orientadas a la producción.

Por otra parte, el presupuesto público que se administra a nivel municipal representa un $33 \%$ de la inversión total nacional. En 2006 las municipalidades rurales manejaron el $70 \%$ de estos recursos, lo que equivale a 2.945 millones de quetzales (aproximadamente 328 millones de dólares). En el cuadro 4 se muestran las inversiones realizadas en 45 municipios pobres localizados en áreas rurales, donde — al igual que en el nivel departamental — predominan las orientadas a la infraestructura. El rubro "otras actividades" incluye los gastos administrativos, a los cuales se destina un alto porcentaje de los recursos recibidos.

Si se toma en cuenta el gasto de los tres niveles de gobierno (central, desconcentrado y descentralizado), en 2006 el gasto público rural fue de 272 dólares per cápita. Esta cifra superó los 90 dólares registrados en Guatemala en 2003 y los 140 dólares per cápita de la media latinoamericana informados por la FAO (Kerrigan, 2001), cuyas estimaciones incluyeron solamente los egresos del gobierno central. Esto implica que las políticas guatemaltecas de desarrollo rural deben considerar el presupuesto de los gobiernos desconcentrado y descentralizado como fuente importante de financiamiento.

\section{La cooperación internacional para el desarrollo rural}

Las donaciones de la cooperación internacional, tanto bilateral como multilateral, equivalen aproximadamente al 2\% del total de gasto rural anual. Su importancia radica en que se trata de asistencia técnica que más tarde alimentará a las políticas públicas.

En 2007, las agencias internacionales de desarrollo habían suscrito un monto total de 282 millones de dólares en proyectos de donación para Guatemala — ejecutables a cinco años plazo-, de los cuales un $78 \%$ correspondía a iniciativas de desarrollo rural (IICA/USAID, 2007a). Los principales rubros de inversión de la cooperación bilateral son el fomento productivo ( $41 \%$ del monto total comprometido), seguido del apoyo a la infraestructura (35\%) y el gasto en salud y recursos humanos (24\%).

La cooperación multilateral no reembolsable, parte de la cual se orienta a las necesidades emergentes del país y el resto a preinversión en proyectos promisorios de crédito, ascendió en 2007 a 251 millones de dólares, de los cuales 140 millones (56\%) correspondieron a

\begin{tabular}{lrrr}
\hline & Quetzales & Dólares & Porcentajes \\
\hline Infraestructura & 239389 & 31499 & 43,0 \\
Gasto social & 139851 & 18401 & 25,0 \\
Fomento productivo & 636 & 84 & 0,1 \\
Otras actividades & 176309 & 23199 & 32,0 \\
Total & 556185 & 73182 & 100,1 \\
\hline
\end{tabular}

Fuente: Instituto Interamericano de Cooperación para la Agricultura/Agencia de los Estados Unidos para el Desarrollo Internacional (IICA/USAID), “Actualización de la base de datos del gasto público rural del período 2002-2006”, Informe de consultoría, IICA-Guatemala, 2007. 
proyectos de desarrollo rural ejecutables a cinco años plazo. Los recursos así obtenidos se destinaron a programas sociales ( $81 \%$ del total), de fomento productivo $(16 \%)$ y de infraestructura $(3 \%)$.

\section{Logros y resultados de las políticas rurales en Guatemala}

En el cuadro 5 se muestran los principales indicadores de avance de las políticas de desarrollo rural. En el ámbito social se amplió la cobertura de hogares con acceso a agua potable, saneamiento, electricidad, salud y educación, indicadores que en el período 1994-2006 crecieron a tasas superiores al 30\%. Por ejemplo, en 2006 el 86\% de los hogares guatemaltecos tenía acceso a agua potable, saneamiento y electrificación, y en el área rural este porcentaje superaba el 75\%. En cuanto a la infraestructura productiva, la red vial creció un $25 \%$ en los últimos 12 años, mientras que las líneas telefónicas se incrementaron más del $1.200 \%$. Por su parte, las exportaciones agrícolas tradicionales como azúcar, café, cardamomo y banano crecieron un $68 \%$ en el período 1994-2006, mientras que las exportaciones agrícolas no tradicionales - frutas, hortalizas y otros - lo hicieron un $255 \%$.

Los resultados de las políticas de desarrollo rural pueden medirse mediante los avances logrados en materia de disminución de la pobreza en el país. A partir del cuadro 6 , en que se muestran los principales

CUADRO 5

Guatemala: principales indicadores de avance de las políticas de desarrollo rural, años 1994, 1998, 2002 y 2006

\begin{tabular}{|c|c|c|c|c|c|}
\hline Indicadores & 1994 & 1998 & 2002 & 2006 & $\begin{array}{c}\text { Crecimiento bruto 1994-2006 } \\
\text { (en porcentajes) }\end{array}$ \\
\hline Acceso a agua potable (en miles de familias) & 1701 & n.d. & 2052 & 2239 & 32,0 \\
\hline Acceso a saneamiento (en miles de familias) & 1447 & n.d. & 1651 & 2.239 & 55,0 \\
\hline Cobertura eléctrica (en miles de familias) & 1095 & 1414 & 1934 & 2.213 & 102,0 \\
\hline Cobertura servicios básicos de salud (en millones de personas) & 0,5 & 2,2 & 3,1 & 4,1 & 720,0 \\
\hline Tasa neta escolaridad primaria (en porcentajes) & 69,0 & 78,0 & 88,0 & 96,0 & 39,0 \\
\hline Acceso a telefonía (en líneas por cada 100 habitantes) & n.d. & 5 & 14 & 66 & $1220,0^{\mathrm{a}}$ \\
\hline Exportaciones agrícolas tradicionales (en millones de dólares) & 633 & 1.229 & 798 & 1.061 & 68,0 \\
\hline Exportaciones agrícolas no tradicionales (en millones de dólares) & 204 & 290 & 424 & 723 & 255,0 \\
\hline
\end{tabular}

Fuente: elaboración propia sobre la base de Banco de Guatemala, "Indicadores económicos de Guatemala" [en línea] http://www.banguat.gob. gt/; Instituto Nacional de Estadística/Secretaría de Planificación y Programación (INE/SEgEPLAN), Necesidades básicas insatisfechas al 2002, Vol. 2, Ciudad de Guatemala, 2006; INE, Encuesta Nacional de Condiciones de Vida (ENCOVI) 2006 [en línea] http://www.ine.gob.gt/ y sEGEPLAN, Avances y desafíos de las políticas públicas en la administración Berger: ejercicio de transición, Ciudad de Guatemala, 2008.

a Representa la tasa de crecimiento del período 1998-2006.

CUADRO 6

Guatemala: pobreza total y pobreza extrema, años 1990,2000 y $2006^{a}$

(En porcentajes y miles de personas)

\begin{tabular}{|c|c|c|c|c|c|c|}
\hline \multirow{2}{*}{ Año } & \multicolumn{3}{|c|}{ Pobreza total } & \multicolumn{3}{|c|}{ Pobreza extrema } \\
\hline & Urbana & Rural & Total pobres (porcentaje) & Urbana & Rural & Total pobres (porcentaje) \\
\hline 2000 & 19 & 81 & $6398(56)$ & 7 & 93 & $1787(16)$ \\
\hline 2006 & 28 & 72 & $6626(51)$ & 17 & 83 & $1977(15)$ \\
\hline
\end{tabular}

Fuente: para 1990: Secretaría de Planificación y Programación (SEGEPLAN), Hacia el cumplimiento de los objetivos de desarrollo del Milenio en Guatemala. II Informe de avances, Ciudad de Guatemala, 2006; para 2000 y 2006: Instituto Nacional de Estadística (INE), Encuesta Nacional de Condiciones de Vida (ENCOVI) 2002 y ENCOVI 2006 [en línea] http://www.ine.gob.gt/.

a Los datos de 1990 corresponden a una medición de la pobreza según indicadores de ingreso, mientras que en 2000 y 2006 se estimó de acuerdo con indicadores de consumo. 
indicadores de pobreza, se aprecia que en el período 1990-2006 la pobreza total se redujo del $61 \%$ al 51\%, mientras que la pobreza extrema disminuyó del $20 \%$ al $15 \%$. Pese al aumento de la pobreza total en términos absolutos, ella decreció cinco puntos porcentuales entre 2000 y 2006.

Al comparar los datos de las zonas rural y urbana, se observa que en el período 2000-2006 la pobreza urbana aumentó del 19\% al 28\%, mientras que la pobreza rural disminuyó del $81 \%$ al $72 \%$. Esto implica que se ha producido una migración de la pobreza desde las áreas rurales hacia las urbanas, principalmente en la categoría de pobreza extrema.

De mantenerse el ritmo actual de reducción de la pobreza, en un escenario optimista Guatemala llegaría a tener un $13 \%$ de pobreza extrema en el año 2015, lo que superaría tres puntos porcentuales el primer objetivo de desarrollo del Milenio (ODM). Fueron precisamente los modestos avances en materia de disminución de la pobreza lo que condujo a que en el país se iniciara un debate sobre las políticas de desarrollo rural, que se analiza en la siguiente sección.

\section{III}

\section{El debate nacional sobre las políticas de desarrollo rural}

Dados los avances en materia de disminución de la pobreza, así como la existencia de algunos aspectos pendientes de los Acuerdos de Paz, en 2002 se inició un proceso de diálogo y discusión sobre la orientación de la política de desarrollo rural. Ese año el gobierno de Alfonso Portillo promovió una mesa intersectorial de diálogo para la formulación de una política nacional sobre el tema, ${ }^{4}$ iniciativa que fue retomada en 2004 por la administración de Óscar Berger. A partir de las discusiones y consensos logrados en las distintas instancias de diálogo, se clarificaron la visión y los objetivos generales de desarrollo rural. ${ }^{5}$ Los principales elementos debatidos se comentan a continuación.

\section{Las orientaciones de política}

En la mesa de diálogo se discutieron principalmente los siguientes temas: i) población-objetivo a la que debían

\footnotetext{
${ }^{4}$ En la Mesa Intersectorial de Diálogo sobre Desarrollo Rural participaron delegados gubernamentales del sector académico, de los partidos políticos, del sector campesino e indígena, de las organizaciones de pequeños productores rurales, de las mujeres rurales, de los gremios empresariales y del sector ambiental.

${ }^{5}$ En la declaración de cierre de la Mesa de Diálogo y Participación de Desarrollo Rural Integral, finalizada en septiembre de 2006, se menciona que "alcanzó importantes avances, entre ellos se consensuaron las principales variables que determinan el desarrollo rural, una visión del área rural a 25 años, los principios y el enfoque que deben orientar la Política de Desarrollo Rural Integral, así como sus ejes de acción" (Gobierno de Guatemala/Gabinete de Desarrollo Rural, 2006, p. 43).
}

orientarse las políticas, ii) importancia del enfoque productivo para complementar la agenda social y iii) aspectos pendientes de la conflictividad agraria. Se definió que el sujeto de las políticas serían los grupos más pobres de las áreas rurales, aquellos que desde la época colonial han sido excluidos y marginados. En este sentido, se identificó como beneficiario final a la pequeña economía rural, que en Guatemala es la indígena y campesina. Desde el punto de vista de las organizaciones sociales del agro, esto significó la oportunidad de fortalecer al campesinado como clase social; para las instancias del Ejecutivo, la posibilidad de focalizar las políticas en los municipios más pobres del país.

El énfasis en la economía campesina implicó también la necesidad de enfocar las políticas en el fomento de la producción rural. Si bien deben continuarse aplicando políticas sociales, es preciso darle prioridad a las inversiones productivas rurales. Según lo discutido en la mesa de diálogo, el objetivo es "dinamizar las economías campesinas". Para estimular la producción se requiere de inversiones públicas estratégicas, por lo que las distintas instancias gubernamentales deben dejar de ser observadores de procesos y convertirse en agentes proactivos de desarrollo rural. Asimismo, en el fomento productivo deben considerarse las ventajas y condiciones locales, de manera que es preciso formular políticas diferenciadas.

En la mesa de diálogo no se logró un consenso generalizado sobre la temática agraria, puesto que las conversaciones se interrumpieron a causa de divergencias 
entre los grupos empresariales y el sector campesino. El planteamiento de las organizaciones campesinas era que el hecho de focalizar las políticas en los grupos más pobres suponía proveerles de activos productivos, siendo la tierra un factor fundamental. Al mismo tiempo, el acceso al recurso tierra implicaba un aumento de la población campesina, con lo cual se perseguía fortalecer al campesinado como clase social. Dado que una de las vías más rápidas para entregar la tierra es la redistribución de la misma, las organizaciones sociales plantearon como principal instrumento de política la expropiación de las fincas mal habidas — por ejemplo, del narcotráfico-y de las tierras ociosas. Sin embargo, el sector empresarial consideró las expropiaciones como una amenaza, pues sentaban precedentes para aplicar una política agraria confiscatoria. El empresariado rural es partidario de formular políticas agrarias orientadas a dinamizar los mercados de tierras, así como de crear instrumentos de desarrollo rural basados en sectores no agrícolas como las artesanías y el ecoturismo.

Mientras se resuelven estas diferencias, la identificación de la economía campesina como sujeto de las políticas contribuiría a dinamizar el mercado de tierras. El desarrollo rural basado en la economía campesina tiene efectos importantes en los mercados de factores. Por ejemplo, el incremento de la productividad agrícola se traduce en una mayor demanda del recurso tierra y, dado que la producción se basa en cultivos de uso intensivo de mano de obra, se tiende a la fragmentación de las fincas medianas y grandes y a la ampliación de las pequeñas, favoreciendo así los cambios en la estructura agraria.

La cuestión agraria es el tema central de lucha ideológica y de clases sociales en las zonas rurales, por lo que se prevé que continuará estando presente en la ruta crítica de su desarrollo (IICA/USAID, 2008). Las discusiones al respecto deben partir de consensos; en este sentido, los avances en cuanto a la definición del sujeto de las políticas y el rol que desempeña el Gobierno en materia de desarrollo rural son puntos de convergencia que permitirían desempantanar el debate sobre política agraria en Guatemala.

\section{Los instrumentos de política}

Si bien en la mesa de diálogo no llegó a establecerse cuáles eran los instrumentos de política adecuados para dinamizar la economía campesina, por tratarse de un asunto técnico se han realizado varios estudios nacionales relacionados con el tema (Barrios y Mellor, 2006; IICA/ USAID, 2008). En primera instancia, la identificación de las economías indígena y campesina como sujeto de las políticas coincide con los planteamientos académicos en cuanto a reducción de la pobreza. En efecto, en el material bibliográfico reciente hay amplias discusiones sobre el potencial de la pequeña agricultura como instrumento para disminuir la pobreza (Banco Mundial, 2007; Mellor, 2007; Christiaensen, Demery y Kühl, 2006; Haggblade, Hazell y Reardon, 2005). El principal argumento es que la agricultura genera un efecto de demanda, en el sentido de que el crecimiento agrícola basado en pequeños productores le genera ingresos a un mayor número de agricultores y asalariados agrícolas, quienes a su vez demandan bienes y servicios agrícolas y no agrícolas en los mercados internos. Ello favorece la creación de empleo urbano y la actividad de los sectores no agrícolas locales, provocando así efectos multiplicadores y círculos virtuosos de disminución de la pobreza (Christiaensen, Demery y Kühl, 2006; Mellor, 2007).

Sin embargo, para que la agricultura sea un instrumento exitoso de reducción de la pobreza deben cumplirse ciertas condiciones mínimas (Haggblade, Hazell y Reardon, 2005; Mellor, 2007), tales como un incremento significativo de la producción de un bien agrícola comerciable. Por otra parte, la mayor productividad de un bien exportable debería generar un aumento considerable de los ingresos locales, lo que se traduce en una distribución masiva y equitativa de las utilidades pertinentes. Esto puede lograrse si la producción se basa en pequeños productores y si los mercados laborales socializan los ingresos, lo cual en Guatemala está vinculado a la economía campesina. Finalmente, es preciso favorecer el surgimiento y consolidación de los sectores no agrícolas, lo que entre otros factores requiere de una institucionalidad pública eficiente y fortalecida que pueda adecuarse a la dinámica de los mercados locales.

En el caso de Guatemala, Barrios y Mellor (2006) e IICA/USAID (2008) identificaron los principales instrumentos para promover el crecimiento económico basado en las economías campesinas, que son compatibles con lo señalado por Allcott, Lederman y López (2006), López (2005) y Fan y Rao (2003) en cuanto al énfasis en los bienes públicos de las políticas de desarrollo. Dichos instrumentos son los siguientes: i) fomento de la investigación y la extensión agropecuaria, ii) creación de infraestructura productiva, principalmente carreteras y sistemas de riego, iii) mejoramiento de la organización productiva y iv) acceso a los mercados financieros.

\section{El enfoque territorial del desarrollo rural}

El enfoque territorial, cuyo fundamento teórico lo proporciona la nueva economía geográfica (Fujita, Krugman y 
Venables, 1999), es un planteamiento exógeno al debate nacional sobre desarrollo rural pero que ha ganado popularidad en Guatemala. Su principal argumento es que las características regionales son las que determinan el desarrollo económico de la región, más que las características propias de las personas (de Ferranti y otros, 2005, pp. 103-124). Los actores económicos se "aglomeran" en territorios específicos con la finalidad de generar condiciones adecuadas para el crecimiento económico (Fujita, Krugman y Venables, 1999). Por otra parte, las regiones ofrecen externalidades positivas que pueden potenciar el desarrollo, lo que también implica que el cierto atraso económico de algunas regiones específicas obedece a la falta de instituciones que "aglomeren" a los actores económicos en torno a la finalidad de aprovechar las ventajas naturales de esos territorios.

De ahí la necesidad de formular políticas diferenciadas regionalmente, con el objetivo de generar mayor equidad entre las distintas regiones de un país dado y optimizar el uso de los recursos (por ejemplo, crear empleos). Estas políticas diferenciadas se originan a partir de una planificación regional en que se logre coordinar a los actores locales en torno a la identificación de políticas y que, al mismo tiempo, pueda atraer los recursos financieros locales necesarios para implementar las acciones priorizadas del consenso social.

Mediante el enfoque territorial pueden alcanzarse los objetivos de política discutidos a nivel nacional, pues permite identificar el potencial productivo de las economías campesinas, convocar a los sectores productivos locales para aunar recursos y buscar objetivos comunes de desarrollo. En este sentido, dicho enfoque encuentra un marco institucional adecuado en las políticas de descentralización y modernización del Estado impulsadas en Guatemala a partir de los Acuerdos de Paz, que se materializaron en la ley de consejos de desarrollo, la ley de descentralización y el nuevo Código Municipal, los cuales se tratan en la siguiente sección.

\section{IV}

\section{La institucionalidad pública formal para el desarrollo rural}

Las orientaciones para la modernización del Estado en Guatemala se basan principalmente en dos fuentes (Sojo, 2000), las cuales en la práctica se han impulsado con diferentes grados de intensidad. Por una parte se encuentran las iniciativas promovidas por la corriente económica de ajuste estructural y apertura comercial, que apuntaron a la reducción del aparato gubernamental y a la eficacia de la política fiscal (incluidos gasto y recaudación). Los principales mecanismos propuestos en esta corriente son el sistema de concesiones, la desincorporación de las empresas estatales y la privatización (Méndez, Gálvez y Vásquez, 2008), así como la descentralización, la menor intervención del Estado y el fomento de la participación ciudadana.

Por otra parte, gracias al proceso de paz en Guatemala se impulsaron acciones positivas encaminadas a crear una sociedad más equitativa, incluyente y participativa. Mediante los Acuerdos de Paz se intentó pasar de un Estado autoritario contrainsurgente hacia uno democrático, incluyente y con una sociedad civil fortalecida. Desde este punto de vista, la descentralización y la participación ciudadana son los instrumentos que permiten que la población se adueñe de su proceso de desarrollo (SEgEPLAN, 2008a).

Puesto que ambas corrientes de pensamiento han tenido influencia en las políticas nacionales, es posible concluir que la modernización del Estado en Guatemala persigue dos grandes objetivos: mejorar la eficiencia estatal en la provisión de bienes y servicios públicos y disminuir la exclusión y la discriminación social (Sojo, 2000; SEGEPLAN, 2008a). Los principales instrumentos para lograr dichos objetivos son la descentralización - o fortalecimiento del poder local—y la creación de mecanismos de diálogo y concertación entre el Estado y la sociedad. ${ }^{6}$

Cabe destacar que Guatemala ha generado un marco legal para lograr los objetivos de modernización del Estado, el cual está representado principalmente por las llamadas leyes de participación y descentralización, a saber: la ley general de descentralización (decreto 14-

\footnotetext{
${ }^{6}$ Recientemente, se ha discutido que la reforma del sistema político es otro elemento indispensable para la modernización del Estado guatemalteco (SEGEPLAN, 2008a).
} 
2002), la ley de los Consejos de Desarrollo Urbano y Rural (decreto 11-2002) y el Código Municipal (decreto 12-2002). ${ }^{7}$

Dicha normativa establece una estructura formal para la formulación, implementación y evaluación de las políticas públicas y, además, prevé la asignación de recursos a los niveles desconcentrado y descentralizado de gobierno. A grandes rasgos, estas leyes definen tres niveles de gobierno (organización vertical), identifican las instancias formales de consenso y concertación —el Sistema de Consejos de Desarrollo Urbano y Rural-y señalan cuáles son las entidades ejecutoras de las políticas públicas (véase diagrama 1).

\footnotetext{
${ }^{7}$ Pese a que las concesiones, desincorporaciones y privatizaciones han tenido relativo éxito en materia de desarrollo rural y administración de los recursos naturales en Guatemala (Méndez, Gálvez y Vásquez, 2008), este documento se enfocará en la institucionalidad formal de descentralización y participación derivada de los Acuerdos de Paz.
}

Desde la perspectiva de ejecución de las políticas públicas, el nivel de mayor jerarquía es el gobierno central, el cual está conformado por el presidente, el vicepresidente y el gabinete de ministros. En el caso de las políticas de desarrollo rural, lo representa el gabinete específico de Desarrollo Rural (GDR) creado en 2006 (acuerdo gubernativo 471-2006). Su objetivo es coordinar las políticas y programas que ejecuta el Ejecutivo en materia de gestión del desarrollo rural y está integrado por siete ministerios y cinco secretarías. En este nivel de gobierno se encuentra también el gabinete Social, que se encarga de coordinar la política nacional pertinente. Así, el GDR tiene un enfoque de promoción (micro) económica, mientras que el gabinete Social está centrado en la educación, la salud y la nutrición. La representación del gobierno central en un gabinete supone que se ha dejado atrás la visión sectorializada del desarrollo para transitar hacia un esquema funcionalmente operativo y de orientación política (Méndez, Gálvez y Vásquez, 2008).

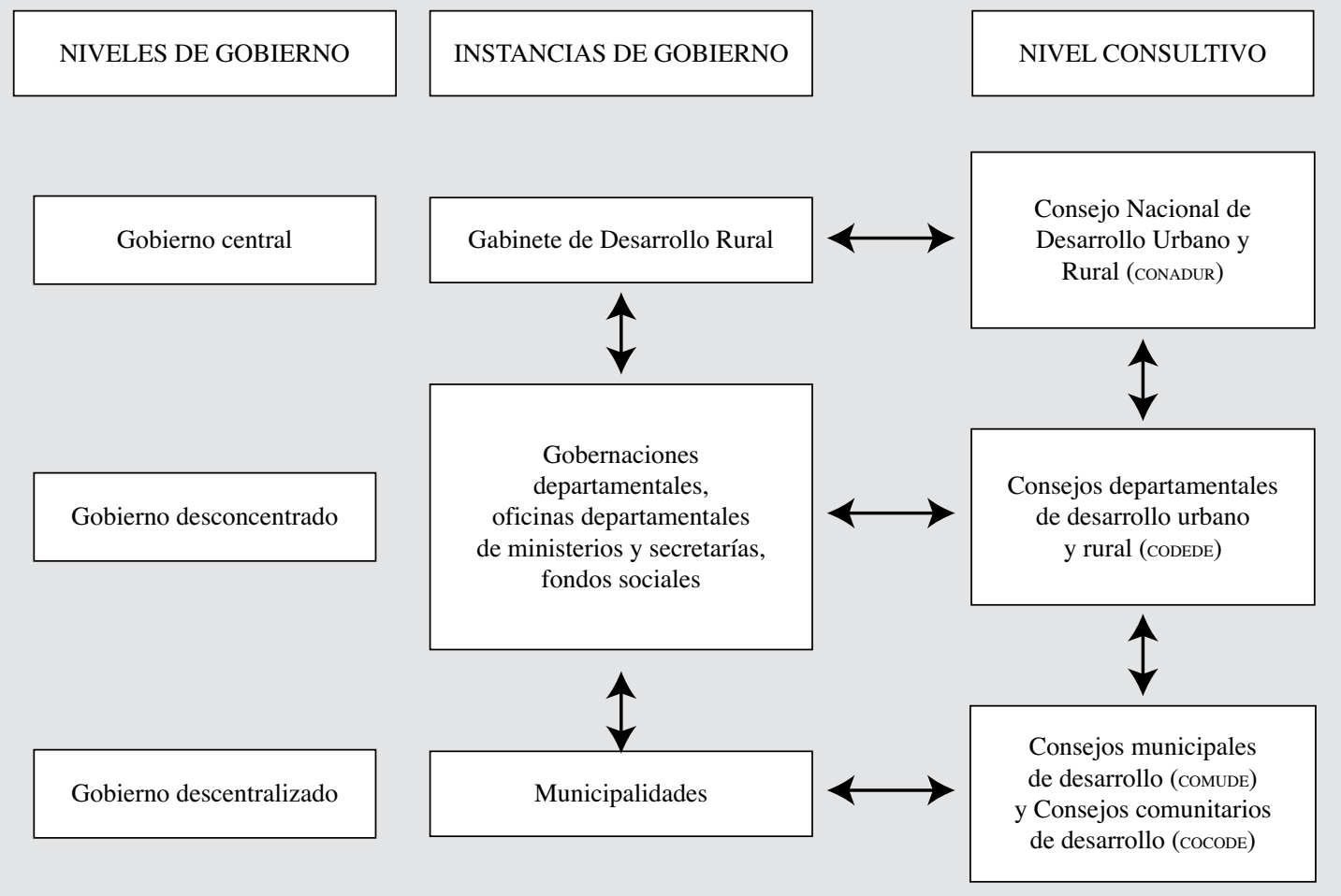

Fuente: elaboración propia sobre la base de leyes de participación y descentralización. 
El carácter del nivel desconcentrado de gobierno es principalmente de planificación y coordinación de acciones en las esferas departamental y territorial, donde los organismos gubernamentales conciertan políticas públicas con los actores sociales y privados. El gobierno desconcentrado está presidido por los gobernadores departamentales, quienes son designados por el Presidente de la República y dependen del Ministerio de Gobernación. En el caso del desarrollo rural, este nivel de gobierno incluye también las oficinas departamentales de los ministerios y secretarías que conforman el gabinete pertinente, así como los fondos de inversión social.

Por su parte, el gobierno descentralizado está representado por los municipios, que tienen autonomía para elegir a sus funcionarios. El fortalecimiento de los gobiernos municipales es el objetivo central de las políticas de descentralización del Estado, tanto en Guatemala como en América Latina. Los municipios cuentan con recursos propios o provenientes de las transferencias del gobierno central y se les han asignado diversas funciones de coordinación y fomento productivo y social.

En cada uno de los niveles de gobierno existen instancias formales específicas para la búsqueda de consensos y la concertación de políticas públicas, llamadas consejos de desarrollo. ${ }^{8}$ Así, el gobierno central cuenta con el Consejo Nacional de Desarrollo Urbano y Rural (CONADUR), que está integrado por representantes de los pueblos indígenas, los campesinos, las mujeres, el empresariado rural y urbano, la academia, las organizaciones no gubernamentales (ONG) de desarrollo y sectoriales, los gobernadores departamentales, los alcaldes y el gabinete de gobierno. La Secretaría de Planificación y Programación de la Presidencia (SEGEPLAN) cumple las funciones de secretaría técnica del gabinete de Desarrollo Rural y del CONADUR, por lo que constituye el enlace para articular el debate entre ambas instancias.

En el nivel desconcentrado o de gobierno departamental las políticas se acuerdan en los consejos departamentales de desarrollo, los cuales están conformados por representantes de los pueblos indígenas, de los

\footnotetext{
${ }^{8}$ Mediante la ley de consejos de desarrollo se creó también el Consejo Regional de Desarrollo Urbano y Rural (COREDUR), pero dado que este no funciona en la práctica, no se discutirá en este documento.
}

grupos de mujeres, de los empresarios urbanos y rurales, de las universidades con presencia en el departamento pertinente y de las oficinas desconcentradas de los ministerios, así como por los alcaldes de cada departamento y el gobernador departamental, quien preside y coordina la instancia. La oficina departamental de la SEGEPLAN es la encargada de la secretaría técnica.

A su vez, el nivel descentralizado o gobierno municipal concierta las políticas en los concejos municipales de desarrollo (COMUDE), que están integrados por los síndicos y concejales de la corporación municipal de que se trate, un máximo de 20 representantes de los consejos comunitarios de desarrollo (COCODE) y representantes de la sociedad y de los ministerios que tengan presencia en el municipio en cuestión. En este caso, la oficina de planificación municipal pertinente es la encargada de la secretaría técnica de los COMUDE.

El marco legal descrito anteriormente regula los recursos financieros asignados a los gobiernos desconcentrado y descentralizado. En el caso del primero, el Estado le transfiere a los consejos departamentales de desarrollo un monto equivalente a un punto porcentual de lo recaudado por concepto de la tasa única del impuesto al valor agregado (IVA), que es del $12 \%$. A los gobiernos municipales les corresponde el $10 \%$ del presupuesto ordinario de ingresos del Estado señalado en la Constitución de la República, 1,5 puntos porcentuales del IVA y diferentes porcentajes del impuesto a la distribución del petróleo, a la circulación de vehículos y de la recaudación central del impuesto único a los bienes inmuebles. Como se mostró en el cuadro 2, los municipios ejecutan el $33 \%$ del presupuesto de inversión pública y los consejos departamentales otro $10 \%$.

Gracias a las políticas de modernización del Estado promovidas mediante los Acuerdos de Paz se ha logrado desarrollar un marco institucional formal para el diseño e implementación de las políticas públicas. Sin embargo, ello no ha permitido que los actores públicos, privados y de la sociedad civil formulen o concierten políticas específicas, ya que si bien dicho espacio es adecuado, todavía no es completamente funcional. En la siguiente sección se analizan los aspectos fundamentales que deben mejorarse para afianzar la institucionalidad de formulación e implementación de políticas públicas en Guatemala, haciendo hincapié en el desarrollo rural. 


\section{V}

\section{Fortalecimiento de la institucionalidad formal para el desarrollo rural}

En la actualidad, no hay una delimitación clara del alcance de las políticas en los tres niveles de gobierno. El marco legal existente no define el tipo de políticas públicas que deben aplicarse en cada uno de ellos, con excepción de las funciones específicas del gobierno municipal. La definición del alcance de las políticas permitirá tener un marco de referencia para la identificación de los elementos relacionados con el fortalecimiento institucional.

En primera instancia, puede argumentarse que al gobierno central le corresponde entregar bienes y servicios públicos nacionales. En este nivel se formulan, por ejemplo, la política de comercio exterior, la política macroeconómica y las políticas de adaptación y mitigación al cambio climático. La bibliografía reciente indica que los gobiernos centrales deben abstenerse de implementar políticas de subsidio de los bienes privados, pues generan clientelismo político (de Ferranti y otros, 2005; López, 2005; Allcott, Lederman y López, 2006) $\mathrm{y}$ afectan el buen funcionamiento de los gobiernos desconcentrado y descentralizado. En el caso del desarrollo rural, el gabinete específico de Desarrollo Rural debe enfocarse en las políticas de investigación y extensión agrícola, la creación de infraestructura — principalmente caminos y sistemas de riego-, el mejoramiento de la organización productiva y la facilitación de los mercados financieros.

El gobierno desconcentrado o departamental debe priorizar las políticas que potencien las ventajas competitivas territoriales. Ejemplos de este tipo de medidas serían la construcción de carreteras que conecten las cabeceras departamentales o de centros de acopio en territorios estratégicos. La identificación de las inversiones a realizar debe efectuarse participativamente entre los distintos actores del territorio, por lo que no existe una definición a priori de las políticas territoriales de desarrollo rural. Pero aun en este caso puede aplicarse el principio de preferir las orientadas a la provisión de bienes y servicios públicos en lugar de subsidiar a sectores específicos (López, 2005; Allcott, Lederman y López, 2006; Schejtman y Berdegué, 2003).

Producto de la descentralización del Estado se les ha entregado a los gobiernos municipales la responsabilidad de proveer servicios públicos a nivel local. El
Código Municipal distingue 15 competencias propias de los municipios, entre ellas la salud, la educación y la seguridad. Los recursos que reciben por derecho constitucional deben invertirse obligadamente en proyectos de educación, salud preventiva, obras de infraestructura y servicios de utilidad pública, mientras que las transferencias originadas a partir del impuesto al valor agregado se destinan a proyectos de infraestructura, educación y salud.

\section{El gobierno central}

El gobierno central puede fortalecerse principalmente en dos aspectos. Primero, es preciso mejorar la eficiencia y eficacia de los instrumentos de política pública y, en segundo lugar, las políticas públicas pueden afianzarse si los actores políticos aprovechan los espacios que brinda el CONADUR para concertar políticas, buscar aliados estratégicos y reducir los costos que supone el diálogo público.

El aumento de la eficiencia y eficacia de las políticas públicas a nivel central puede lograrse priorizando el gasto público en los territorios más pobres de las áreas rurales y focalizándose además en las economías campesinas. La eficacia de las políticas pasa también por reducir el gasto en subsidios privados y aumentar los instrumentos orientados a fomentar la provisión de bienes y servicios públicos. Para esos efectos se requiere una coordinación al más alto nivel político.

En varios estudios recientes (Banco Mundial, 2003; Vergara y Lavarreda, 2006) se ha abordado el tema de eficiencia del gasto social en Guatemala. Al respecto, el Banco Mundial (2003) sostuvo que la incidencia del gasto público en los programas de protección social fue regresiva, puesto que el quintil más rico recibió el $46 \%$ de la inversión social y el quintil más pobre únicamente el $8 \%$. En este estudio se concluyó que ninguno de los programas de protección social estaba bien focalizado, pues la atención hacia los sectores pobres era reducida.

Dado que en Guatemala la pobreza rural está geográficamente ubicada - la mayoría de los pobres vive en el altiplano nacional-, la alternativa de política es 
focalizar los programas sociales en esos territorios del país (Banco Mundial, 2003). En este sentido, aunque ya se han aplicado algunos programas como Guate Solidaria Rural y la Estrategia de reducción de la desnutrición crónica — que se iniciaron en 2005- y más recientemente el Programa de transferencias condicionadas —iniciado en el segundo semestre de 2008_, es fundamental continuar focalizando los recursos en los territorios y poblaciones pobres para que el gasto social rural tenga mayores efectos en materia de reducción de la pobreza.

Además de lo anterior, sería necesario darle prioridad a los instrumentos de política cuyo objetivo sea estimular la provisión de bienes públicos. El gasto en fomento productivo agrícola, por ejemplo, se compone sobre todo de programas de fertilizantes y del programa Bosques y Agua para la Concordia —este último es una retribución a las patrullas de autodefensa civil por su participación en el conflicto armado-, que se clasifican claramente como subsidios privados. Si disminuyera este tipo de subsidios y se incrementara la inversión en bienes públicos, se lograría aumentar el crecimiento agrícola (López, 2005; Allcott, Lederman y López, 2006).

La focalización de las políticas en las poblaciones y territorios pobres, así como en la provisión de bienes públicos, requiere de una coordinación institucional al más alto nivel político. En Guatemala se han aplicado diversas medidas al respecto, tales como la designación por el Presidente de comisionados gubernamentales que coordinan a los ministros de Estado, los consejos o comisiones interinstitucionales —en que una junta colegiada de instancias gubernamentales y privadas lleva a cabo la coordinación-y los gabinetes específicos de gobierno, conformados por un número reducido de ministerios y secretarías y presididos por el vicepresidente. Dado que estos últimos son la única figura reconocida en la estructura institucional (SEGEPLAN, 2008a, p. 211), se recomienda continuar fortaleciéndolos para aumentar la capacidad gubernamental de coordinación, supervisión y evaluación de las políticas públicas.

El interés del gobierno central por el CONADUR radica en dos aspectos. Primero, el Sistema de Consejos de Desarrollo Urbano y Rural es una estructura adecuada para buscar coherencia y correspondencia entre las políticas nacionales y las descentralizadas. En segundo lugar, el CONADUR permite agilizar la concertación y negociación de ellas, disminuyendo los costos de transacción que implica el diálogo pertinente y, además, generando aliados para la implementación de las medidas. Es preferible tener un solo interlocutor formal que numerosas mesas de discusión.
Una experiencia reciente en los intentos por vincular las políticas locales a las políticas nacionales fue el Reglamento para la administración del aporte a los CODEDE (IICA/USAID, 2008), aprobado por el CONADUR en diciembre del 2007. Mediante esta disposición legal se crearon incentivos económicos para los consejos departamentales, cuyas inversiones se alinearan con las políticas públicas, y se fijaron criterios normativos a cumplir en los proyectos que emprendieran los diversos departamentos. El CONADUR, como ente político de concertación, desempeñó un papel importante en la aprobación de este Reglamento.

Por su parte, la sociedad civil puede canalizar sus demandas e influir en la toma de decisiones mediante los espacios institucionalmente conformados. Por ejemplo, puede desempeñar un papel importante en la reducción de los subsidios privados, aprovechando la posibilidad que le ofrecen los consejos de desarrollo de actuar como fiscalizadora de las políticas públicas. Sin embargo, la utilización del espacio que les brinda el CONADUR es una decisión política que los actores sociales han subestimado.

\section{El gobierno desconcentrado}

La principal debilidad del gobierno desconcentrado fue señalada por la SEGEPLAN: "los procesos para propiciar la participación, profundizar la descentralización y fortalecer el poder local se han dado de forma desordenada, privilegiando la descentralización financiera sin reglas claras. Con ello se ha favorecido que la gestión municipal y la gestión que realizan los consejos de desarrollo - con financiamiento transferido por el gobierno central-esté desarticulada de las políticas públicas" (sEgEPLAN, 2008a, p. 219). El nivel desconcentrado de gobierno es aquel en que se vinculan las políticas locales con las nacionales, siendo además el espacio en que se planifican e implementan las políticas de desarrollo rural.

El enfoque territorial del desarrollo rural cobra sentido a nivel departamental. Ello se aprecia en más de 22 ejercicios de planificación territorial desarrollados en Guatemala, que probaron ser instrumentos eficaces de gestión pública para orientar los procesos de participación, descentralización y fortalecimiento del poder local (SEGEPLAN, 2008a). Es por ello que las estrategias encaminadas a fortalecer el nivel desconcentrado de gobierno se centran en el proceso de planificación territorial.

En una evaluación de la planificación territorial realizada por el Instituto Interamericano de Cooperación para la Agricultura/Agencia de los Estados Unidos para el 
Desarrollo Internacional (IICA/USAID) (2007b), se sostuvo que el principal reto de los CODEDE no era mejorar los aspectos técnicos de la planificación, que siempre serán perfectibles, sino aprovechar las distintas etapas de ella - es decir, diálogo, concertación y negociación- para movilizar los recursos públicos y privados a fin de alcanzar objetivos comunes de desarrollo. Esto implica, por una parte, una fase de negociación y concertación de los objetivos y la visión territorial de los principales actores locales y, por otra, orientar los recursos financieros de la sociedad civil, el sector privado y el Gobierno hacia las acciones y proyectos negociados y concertados en el proceso de planificación. En otras palabras, la planificación estratégica debe convertirse en un pacto político-social entre los actores locales para transformar el territorio de que se trate.

Para lograr que la planificación territorial se convierta en un pacto político-social se requiere ampliar la participación de los actores locales, principalmente de la sociedad civil y del sector privado, puesto que al definir las acciones predomina la presencia de las instancias del Estado. En la planificación territorial como proceso de concertación política deben identificarse medidas concretas, responsabilidades y plazos, utilizándolos como instrumento de presión social y política para que los actores pertinentes cumplan los acuerdos comprometidos en ese contexto. Para ello, el IICA/USAID (2007b) recomendó impulsar la creación de mecanismos legales tales como los contratos territoriales, en que se indiquen las sanciones a que se exponen los actores en caso de incumplimiento de sus compromisos.

El mayor reto de la planificación territorial a nivel departamental es vencer la institucionalidad informal heredada de 36 años de guerra civil, en que el denominador común de los actores locales ha sido la renuencia a participar en los procesos de desarrollo. Esto podría lograrse garantizando que lo pactado en los procesos de planificación se refleje en proyectos de inversión. De ahí la importancia que tiene vincular la planificación territorial a la elaboración del presupuesto del Estado, lo cual todavía no se ha concretado: la mayoría de los proyectos identificados en los procesos de planificación territorial no se reflejan en el presupuesto de inversión de los departamentos. Los gobiernos departamentales deben ser capaces de concertar los acuerdos políticos necesarios para canalizar recursos hacia las acciones territoriales. ${ }^{9}$

\footnotetext{
${ }^{9}$ Existe inquietud en torno a las posibilidades reales de los gobernadores departamentales de lograr acuerdos políticos a nivel territorial. Por ejemplo, el gobernador del departamento de Huehuetenango, designado
}

En síntesis, las principales funciones del gobierno desconcentrado son coordinar y planificar las políticas públicas departamentales. El enfoque territorial es un instrumento de probada eficacia en la identificación y definición del orden de prioridades de la inversión pública. Sin embargo, debe ampliarse su alcance para incluir a un mayor número de actores sociales y privados, así como convertir la planificación territorial en un pacto político-social que cuente con los mecanismos necesarios para incentivar la implementación de las acciones. Ello permitiría asegurar la sostenibilidad del enfoque territorial del desarrollo rural y, al mismo tiempo, afianzar la institucionalidad formal de participación ciudadana. El reto está en vencer la renuencia existente al respecto, lo que se logrará si los pactos políticos se aplican realmente en el terreno. Se prevé que la planificación territorial a nivel departamental puede ser un instrumento adecuado para el desarrollo rural, que contribuye también a la modernización del Estado en su nivel desconcentrado.

\section{El gobierno descentralizado y la cooperación internacional}

En diversos estudios sobre la gestión municipal (por ejemplo Letelier, 2007) se han identificado los siguientes temas sustantivos del municipalismo: vincular el gasto municipal a las políticas públicas, aumentar el grado de recaudación de los municipios y mejorar el tipo de inversiones que estos realizan. También es indispensable ampliar las capacidades municipales de planificación. Además, el fortalecimiento de la participación ciudadana en la concertación y negociación política es un factor que contribuiría a perfeccionar los procesos democráticos nacionales. Dado que el vínculo entre la política nacional y los intereses locales se materializa a nivel departamental, sería importante fortalecer esta instancia de gobierno para mejorar la eficacia de las políticas municipales.

Por su parte, la cooperación internacional puede desempeñar un papel activo en la consolidación de la institucionalidad formal para el desarrollo rural. Su contribución más importante, sea productiva, participativa o circunstancial, sería proporcionar asesoría técnica para que en la formulación y concertación de las políticas públicas se incorpore lo planificado a nivel local, así como el fortalecimiento de la organización civil.

por el Presidente, tiene poco respaldo político para negociar con los 31 alcaldes y 10 diputados distritales elegidos democráticamente. 


\section{VI}

\section{Conclusiones}

El comportamiento de las políticas de desarrollo rural en Guatemala pareciera ser similar al de una orquesta desafinada y descoordinada: existen recursos financieros considerables — superiores a la media latinoamericana-, pero han tenido escasos efectos en materia de disminución de la pobreza. Esta aseveración es válida para los niveles central, desconcentrado y descentralizado de gobierno. El debate reciente sobre las políticas de desarrollo rural indica que una primera medida sería fortalecer la institucionalidad de formulación, concertación e implementación de políticas públicas.

Mediante los Acuerdos de Paz se propuso una estructura institucional que se adaptara a los nuevos lineamientos de desarrollo rural, al crear una institucionalidad lógica que promueve la participación ciudadana y la concertación de políticas públicas. Sin embargo, aunque el marco legal está dado, en la práctica los actores políticos y sociales "no juegan en esa cancha". En este artículo se identifica una serie de medidas que sería preciso aplicar en los tres niveles de gobierno para fortalecer la institucionalidad de la paz y el desarrollo rural, las cuales pueden impulsar los actores públicos, privados o sociales.

A nivel central, los ciudadanos tendrían mayores posibilidades de hacer que sus propuestas fueran vinculantes si participaran efectivamente en el CONADUR, en lugar de hacerlo en "mesas de diálogo" cuyas conclusiones no llegan después a concretarse en políticas públicas. Mediante la participación ciudadana en el CONADUR, estas últimas deberían reorientarse a la inversión en bienes públicos - los cuales han sido identificados en la discusión nacional- y a eliminar los mecanismos clientelistas que favorecen a grupos de presión específicos. Por su parte, el gobierno central podría agilizar el proceso de concertación de políticas si dialoga con un solo interlocutor (el CONADUR), disminuyendo los costos de negociación y concertación a la vez que genera aliados para su implementación.

El nivel departamental de gobierno es quizás el que más requiere de fortalecimiento y aquel en que el enfoque territorial de desarrollo tiene mayores repercusiones. $\mathrm{Al}$ respecto, lo principal es hacer que lo concertado a nivel departamental se traduzca en políticas públicas. Esto implica que todos los actores deben tener compromisos y metas claramente establecidas en los planes de desarrollo territorial. Los contratos público-sociales que se han impulsado en algunos países de América Latina podrían ser el mecanismo adecuado para lograr dicho objetivo y, además, para aumentar el financiamiento de las inversiones territoriales en bienes públicos.

Los gobiernos municipales (nivel descentralizado) todavía necesitan mejorar la capacidad técnica de las oficinas de planificación. Igualmente importante es continuar promoviendo la participación ciudadana en la fiscalización y formulación de las políticas públicas. El enfoque territorial de desarrollo ha probado ser un medio eficaz para generar políticas y aunar los esfuerzos de los distintos actores sociales a nivel local.

Al promover políticas territoriales dentro del marco formal existente, se estaría asegurando una mayor sostenibilidad de las políticas de desarrollo rural. Al mismo tiempo, se fortalecería la institucionalidad formal de participación e inclusión, pues esta tendría objetivos claros que justifican su existencia: priorizar las inversiones públicas y privadas. Con ello se contribuiría también a la modernización del Estado guatemalteco, condición indispensable para lograr un desarrollo verdadero y la inserción exitosa de Guatemala en la economía del siglo XXI.

\section{Bibliografía}

Allcott, H., D. Lederman y R. López (2006), "Political institutions, inequality, and agricultural growth: the public expenditure connection", Policy Research Working Paper, No 3902, Washington, D.C., abril.

Banco de Guatemala (2008), "Indicadores económicos de Guatemala" [en línea] http://www.banguat.gob.gt/.

Banco Mundial (2007), Informe sobre el desarrollo mundial 2008: agricultura para el desarrollo, Washington, D.C.
(2004), "Drivers of sustainable rural growth and poverty reduction in Central America. Guatemala case study", World Bank Report, № 31191-GT, Washington, D.C.

(2003), "La pobreza en Guatemala", serie Estudios del Banco Mundial sobre países, $\mathrm{N}^{\circ} 27586$, Washington, D.C.

Barrios, J.M. y J. Mellor (2006), "Distribución sectorial del crecimiento del empleo en el altiplano guatemalteco", Documentos técnicos, $\mathrm{N}^{\mathrm{o}} 17$, Ciudad de Guatemala, Universidad Rafael 
Landívar/Instituto de Agricultura, Recursos Naturales y Ambiente (IARNA).

Christiaensen, L., L. Demery y J. Kühl (2006), “The role of agriculture in poverty reduction. An empirical perspective", Policy Research Working Paper Series, N ${ }^{\circ}$ 4013, Washington, D.C., Banco Mundial.

De Ferranti, D. y otros (2005), Beyond the City. The Rural Contribution to Development, Washington, D.C., Banco Mundial.

Ellis, F. (2000), Rural Livelihoods and Diversity in Developing Countries, Nueva York, Oxford University Press.

Fan, S. y N. Rao (2003), "Public spending in developing countries: trends, determination, and impact", EPTD Discussion paper, $\mathrm{N}^{\circ} 99$, Washington, D.C., Instituto Internacional de Investigaciones sobre Políticas Alimentarias.

Fujita, M., P. Krugman y A. Venables (1999), The Spatial Economy: Cities, Regions and International Trade, Cambridge, Massachusetts, The MIT Press.

Gobierno de Guatemala/Gabinete de Desarrollo Rural (2006), Política de desarrollo rural, Ciudad de Guatemala.

Gobierno de Guatemala/Secretaría de la Paz (2007), Situación actual del cumplimiento de los Acuerdos de Paz 2004-2006 (Enfoque temático), Ciudad de Guatemala, PNUD-Guatemala.

Gobierno de Guatemala/Unidad Revolucionaria Nacional Guatemalteca (1996), Acuerdo sobre Aspectos Socioeconómicos y Situación Agraria [en línea] http://www.congreso.gob.gt/Docs/paz/ acuerdosobreaspectossocioeconomicosysituacionagraria.pdf

Haggblade, S., P. Hazell y T. Reardon (2005), "The rural nonfarm economy: pathway out of poverty of pathway in?", The Future of Small Farms. Proceedings of a Research Workshop (Wye, Reino Unido, 26 a 29 de junio).

IICA/USAID (Instituto Interamericano de Cooperación para la Agricultura/ Agencia de los Estados Unidos para el Desarrollo Internacional) (2008), Informe final del Convenio de Cooperación USAID/IICA $N^{o}$ 520-A-00-05-0096-00, Ciudad de Guatemala.

(2007a), "Actualización de la base de datos de los temas de intervención y aportes financieros de la cooperación internacional en el área rural de Guatemala", Informe de consultoría, IICAGuatemala.

(2007b), "Evaluación del modelo de gestión territorial de Guatemala", Informe de consultoría, IICA-Guatemala.

(2007c), "Actualización de la base de datos del gasto público rural del período 2002-2006”, Informe de consultoría, IICA-Guatemala.

INE (Instituto Nacional de Estadística) (2007), Encuesta Nacional de Condiciones de Vida (ENCOVI) 2006, Ciudad de Guatemala [en línea] http://www.ine.gob.gt/.

(2002), Encuesta Nacional de Condiciones de Vida (ENCOVI) 2000, Ciudad de Guatemala.

INE/SEGEPLAN (Instituto Nacional de Estadística/Secretaría de Planificación y Programación) (2006), Necesidades básicas insatisfechas al 2002, vol. 2, Ciudad de Guatemala.

Kerrigan, G. (2001), Gasto público hacia el sector agrícola y desarrollo de las áreas rurales: ALC, tendencias y desafios, Santiago de
Chile, Organización de las Naciones Unidas para la Agricultura y la Alimentación (FAO).

Letelier, L. (2007), "El gasto municipal en Centroamérica y República Dominicana", Serie de estudios económicos y sectoriales, $\mathrm{N}^{\circ}$ RE2-07-006, Washington, D.C., Banco Interamericano de Desarrollo.

López, R. (2005), "Under-investing in public goods: evidence, causes, and consequences for agricultural development, equity and the environment", Agricultural Economics, vol. 32, s.1, International Association of Agricultural Economists.

Mellor, J. (2007), "Temas fundamentales para la reducción de la pobreza: la interacción de la inclusión social, el desarrollo agrícola y el gasto social", ponencia presentada en el Simposio de desarrollo sobre la inclusión social y la erradicación de la pobreza, Ciudad de Guatemala.

Méndez, J.C., J. Gálvez y E. Vásquez (2008), "La modernización institucional, base para la definición y aplicación eficiente de políticas", Políticas de recursos naturales en Centroamérica: lecciones, posiciones y experiencias para el cambio, R. de Camino, A. Ballestero y J. Breitling (eds.), San José, Universidad para la Paz.

MINUGUA (Misión de Verificación para Guatemala) (2004), Retomando el camino: tareas pendientes en la construcción de la paz. Situación socioeconómica y desarrollo rural. Informe de MINUGUA, Ciudad de Guatemala.

Schejtman, A. y J. Berdegué (2003), "Desarrollo territorial rural", Desarrollo territorial rural en América Latina y el Caribe: manejo sostenible de recursos naturales, acceso a tierras y finanzas rurales, R.G. Echeverría, Washington, D.C., Banco Interamericano de Desarrollo.

SEgEPLAN (Secretaría de Planificación y Programación) (2008a), Avances y desafios de las políticas públicas en la administración Berger: ejercicio de transición, Ciudad de Guatemala.

(2008b), "Actualización de la base de datos del gasto público rural del período 2006-2007", Informe de consultoría, Ciudad de Guatemala.

(2007), "Análisis del comportamiento del aporte a los consejos departamentales de desarrollo en el marco de las orientaciones de política período 2004-2007”, Ciudad de Guatemala, Dirección de Inversión Pública, inédito.

(2006), Hacia el cumplimiento de los objetivos de desarrollo del Milenio en Guatemala. II Informe de avances, Ciudad de Guatemala.

Sepúlveda, S. y otros (2003), El enfoque territorial del desarrollo rural, San José, Instituto Interamericano de Cooperación para la Agricultura.

Sojo, C. (2000), "El traje nuevo del emperador: la modernización del Estado en Centroamérica", Documento de trabajo, $\mathrm{N}^{\circ} 6$, Hamburgo, Institut für Iberoamerika-Kunde.

Vergara, R. y J. Lavarreda (2006), "El gasto público en Guatemala", Serie de estudios económicos y sectoriales, $\mathrm{N}^{\circ} \mathrm{RE2}-06-032$, Washington, D.C., Banco Interamericano de Desarrollo. 